\title{
Stability of the Wurtzite Structure
}

\author{
Lawætz, Peter
}

Published in:

Physical Review B Condensed Matter

Link to article, DOI:

10.1103/PhysRevB.5.4039

Publication date:

1972

Document Version

Publisher's PDF, also known as Version of record

Link back to DTU Orbit

Citation (APA):

Lawætz, P. (1972). Stability of the Wurtzite Structure. Physical Review B Condensed Matter, 5(10), 4039-4045. https://doi.org/10.1103/PhysRevB.5.4039

\section{General rights}

Copyright and moral rights for the publications made accessible in the public portal are retained by the authors and/or other copyright owners and it is a condition of accessing publications that users recognise and abide by the legal requirements associated with these rights.

- Users may download and print one copy of any publication from the public portal for the purpose of private study or research.

- You may not further distribute the material or use it for any profit-making activity or commercial gain

- You may freely distribute the URL identifying the publication in the public portal

If you believe that this document breaches copyright please contact us providing details, and we will remove access to the work immediately and investigate your claim. 


\title{
Stability of the Wurtzite Structure \\ Peter Lawaetz \\ Physics Laboratory III, Technical University of Denmark, DK-2800 Lyngby, Denmark \\ (Received 17 September 1971)
}

\begin{abstract}
An analysis of available data for 20 wurtzite compounds of the $A^{N} B^{8-N}$ type shows that the stability as compared with zinc blende is closely connected with deviations of the $c / a$ ratio from the ideal value of 1.633. A simple qualitative model is proposed to account for this feature. The variation in $c / a$ is then correlated with the charge parameter $Z C / \hbar \omega_{p}$, where $Z$ is the (effective) valence, $C$ Phillips's electronegativity difference, and $\hbar \omega_{p}$ the plasma energy of the free-valence-electron gas. The results indicate that $c / a$ may be predicted with an uncertainty of $0.1 \%$.
\end{abstract}

\section{INTRODUCTION}

Tetrahedrally coordinated binary compounds of the $A^{N} B^{8-N}$ type are found in either the cubic zincblende $(B)$ or the hexagonal wurtzite $(W)$ structure or sometimes even in both modifications. While the ionicity scale recently proposed by Phillips ${ }^{1}$ and Van Vechten ${ }^{2}$ adequately describes the transition from fourfold to sixfold coordination and possibly also to eightfold coordination, it is not able to distinguish clearly between structures having the same coordination. ${ }^{2}$ This is not too surprising because ionicity is a measure of the character of the bond and essentially a short-range property, whereas the $B$ and $W$ structures differ mainly in the relative position of third neighbors and beyond (see Fig. 1) so that long-range interactions are important here.

In addition to the difference in the configuration of distant neighbors, the lower symmetry of the $W$ structure allows for a distortion along the $c$ axis away from the regular tetrahedral bond distribution. Thus the $c / a$ ratio deviates slightly from its ideal value of $\left(\frac{8}{3}\right)^{1 / 2} \approx 1.633$, and the $u$ parameter (ratio of nearest-neighbor distance along the $c$ axis to $c$ ) similarly from $\frac{3}{8}=0.375$.

In this paper we want to point out the existence of a close correlation between the sign and magnitude of the $c / a$ deviation on one hand, and the stability of the $W$ structure on the other. It turns out that this puzzling feature can be understood qualitatively, but the limited knowledge about long-range interactions prohibits a detailed theory. We then show that experimental values of the $c / a$ ratio are correlated with the parameter $\left(Z C / \hbar \omega_{p}\right)^{2}$, where $Z$ is the valence, $C$ is Phillips's electronegativity difference, ${ }^{1}$ and $\hbar \omega_{p}$ is the plasma energy of the valence-electron gas. ${ }^{1}$ In this way, also the $W$ vs- $B$ stability can be described by quantities derived from the ionicity concepts introduced by Phillips ${ }^{1}$ and Van Vechten. ${ }^{2}$

It is generally known that the Madelung constant for the ideal $W$ configuration is about $0.2 \%$ larger than that of $B$ so that considerations of the ionic energy favor $W$. Keffer and Portis ${ }^{3}$ showed that a decrease in $c / a$ would increase the Madelung constant still further so that wurtzites should have $c / a<1.633$. Jeffrey, Parry, and Mozzi ${ }^{4}$ had already noticed this feature in the limited experimental data available at that time. Since Keffer and Portis ${ }^{3}$ neglected nonionic long-range effects completely, their theory of the distortion does not give solutions with $c / a>1.633$ although several compounds occur in this region. The theory is therefore subject to serious limitations and does not elucidate the problem of stability.

Van Vechten ${ }^{2}$ has proposed an entirely different explanation for the stability. Based on some side effects of his otherwise excellent "dielectric" bandstructure calculation, he suggested that a material could not tolerate having its valence band edge at the $X$ point rather than at $\Gamma$ in the Brillouin zone. Apart from giving a questionable borderline between the two structures, this proposal is not clear because the point in the $W$ structure corresponding

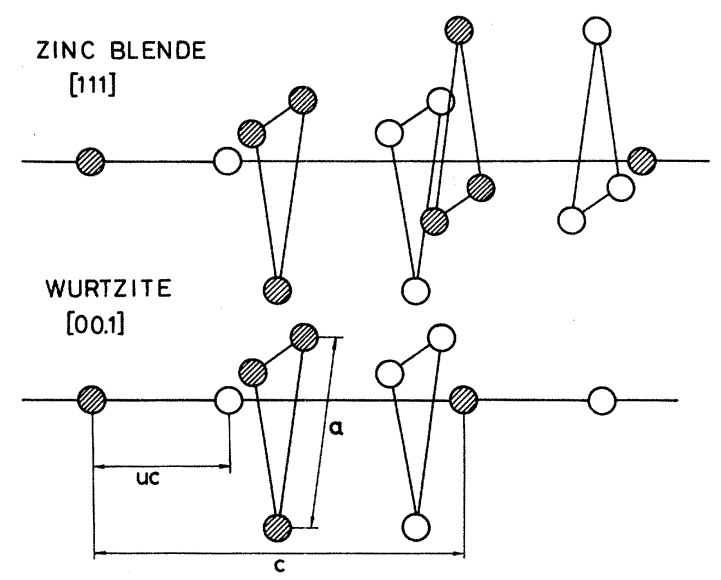

FIG. 1. Comparison of atomic configuration in zinc blende and wurtzite along the trigonal axis. The wurtzite lattice parameters $a, c$, and $u$ are indicated. 
TABLE I. Collection of wurtzite lattice parameters for $A^{N} B^{8-N}$ compounds.

\begin{tabular}{lllll}
\hline Crystal & $a(\AA)$ & $c / a$ & \multicolumn{1}{c}{$u$} & Reference \\
\hline AlN & 3.111 & 1.600 & 0.385 & 4 \\
$\mathrm{ZnO}$ & 3.250 & 1.602 & 0.3826 & $\mathrm{a}, \mathrm{b}$ \\
$\mathrm{InN}$ & 3.533 & 1.611 & & $\mathrm{c}$ \\
$\mathrm{MgS}$ & 3.95 & 1.62 & & $\mathrm{~d}$ \\
$\mathrm{MgSe}$ & 4.145 & 1.622 & & 18 \\
$\mathrm{GaN}$ & 3.160 & 1.622 & & $\mathrm{e}$ \\
$\mathrm{BeO}$ & 2.698 & 1.623 & 0.3785 & $4, \mathrm{~b}$ \\
$\mathrm{CdS}$ & 4.136 & 1.623 & & 20 \\
$\mathrm{MgTe}$ & 4.53 & 1.629 & & $\mathrm{f}$ \\
$\mathrm{CdSe}$ & 4.299 & 1.631 & & $\mathrm{~g}$ \\
$\mathrm{Ideal}$ & $\cdots$ & 1.633 & 0.3750 &. \\
$\mathrm{ZnSe}$ & 4.003 & 1.634 & & $\mathrm{~h}$ \\
$\mathrm{AgI}$ & 4.592 & 1.635 & 0.3747 & 7 \\
$\mathrm{ZnS}$ & 3.823 & 1.637 & & $\mathrm{i}$ \\
$\mathrm{CdTe}$ & 4.572 & 1.637 & & $\mathrm{j}$ \\
$\mathrm{CuBr}$ & 4.06 & 1.640 & & 5 \\
$\mathrm{SiC}$ & 3.076 & 1.641 & & $\mathrm{k}$ \\
$\mathrm{CuCl}$ & 3.91 & 1.642 & & 6 \\
$\mathrm{BN}$ & 2.55 & 1.645 & & 24 \\
$\mathrm{CuI}$ & 4.31 & 1.645 & & 5 \\
$\mathrm{ZnTe}$ & 4.310 & 1.645 & & 1 \\
\hline \hline
\end{tabular}

${ }^{2}$ S. C. Abrahams and J. L. Bernstein, Acta Cryst. B 25,1233 (1969)。

T. M. Sabine and S. Hogg, Acta Cryst. B $\underline{25}, 2254$ (1969).

${ }^{\circ} R$. Juza and H. Hann, Z. Anorg. Allgem. Chem. 239, 282 (1938).

${ }^{\mathrm{d}} \mathrm{G}$. Berthold, Z, Physik 181, 333 (1964).

${ }^{\mathrm{e}} \mathrm{G}$. V. Lirman and G. S. Zhdanov, Acta Physicochim. URSS $\underline{6}, 306$ (1937).

${ }^{\mathrm{f}} \mathrm{W}$. Klemm and K. Wahl, Z. Anorg. Chem. 266, 289 (1951).

${ }^{\mathrm{H}} \mathrm{H}$ E. Swanson, N. T. Gilfrich, and M. I. Cook, Natl. Bur. Std. (U. S。), Circ. No. 539, 7, 12 (1957).

${ }^{\text {h}} Y$. S. Park and F。L. Chan, J. Appl. Phys. 36, 800 (1965).

${ }^{1}$ B. J. Skinner and P. M. Bethke, Am. Mineralogist 46,1382 (1961).

${ }^{j}$ I. Spinulescu-Carnaru, Phys. Status Solidi 15, 761 (1966).

${ }^{\mathrm{k}} \mathrm{R} . \mathrm{F}$. Adamsky and K. M. Merz, Z. Krist. 111, 350 (1959).

${ }^{1}$ I. Spinulescu-Carnaru, Phys. Status Solidi 18, 769 (1966).

to $X$ (in the $B$ structure) is still out in the Brillouin zone. In addition, Van Vechten's model leads to a valence band edge at $\Gamma$ originating from the $L$-point in the $B$ structure, whereas experimental results have been accounted for by a quasicubic model based on the $p$ states of the original $\Gamma$ valence band edge. Furthermore, the idea presupposes that there is no energy difference at all between the $W$ and $B$ structure, whereas it will be shown here that the important difference may generally be of the order $0.2 \%$ as in the Madelung constants, corresponding to an average difference of about $10 \mathrm{meV}$ per valence electron. There is little hope that over-all band- structure calculations will attain such an accuracy.

\section{STABILITY AND $c / a$ RATIO}

Twenty $A^{N} B^{8-N}$ compounds have definitely been shown to occur with the $W$ structure. Their principal lattice parameters $a$ and $c$ have been measured by standard $\mathrm{x}$-ray, neutron, or electron diffraction techniques and in most cases they are known with an appreciable accuracy. However, it will be apparent from the present analysis that the $c / a$ ratio is very sensitive to crystal imperfections, thermal stresses, and impurity content, and so some materials, notably MgS, ZnS, and CdS, exhibit a relatively large spread in the experimental $c / a$. The data shown in Table I are therefore the author's selection based on a number of criteria such as general trends, minimum volume difference between $W$ and $B$ modifications, etc. It is seen from Table I that there are just as many compounds having $c / a>1.633$ as there are with $c / a<1.633$, and so the importance of the former region is obvious.

It is not straightforward to decide from available experiments whether a certain compound is stable in the $W$ or in the $B$ modification. Of the $20 \mathrm{ma-}$ terials listed in Table I, twelve also occur in $B$ versions, two in the rocksalt structure ( $\mathrm{MgS}$, $\mathrm{MgSe}$ ), and $\mathrm{Mg} \mathrm{Te}$ and InN crystals are very difficult to grow. This leaves four real $W$ crystals: AlN, GaN, BeO, and $\mathrm{ZnO}$. These have all appreciable negative $\Delta(c / a)=c / a-1.633$. The other extreme with $\Delta(c / a)>0$ is represented by such materials as $\mathrm{ZnTe}$ and $\mathrm{CuI}$ which must be considered as stable $B$ crystals since very few are even aware of the existence of a $W$ form. For $c / a$ near ideal, it is much more difficult to assess the real zerotemperature stability of the materials in question, and this is of course the first indication of the special nature of the ideal ratio in relation to stability. Here we have been guided by two experimental features as described in the following.

For the copper and silver halides, there is a definite transition temperature between hexagonal and cubic, ${ }^{5,6}$ the latter being the preferred low-temperature structure. Thus, they are all definitely stable $B$ crystals in our sense, and all have $\Delta(c / a)>0$. Note that AgI have only an extremely small distortion $[\Delta(c / a)=+0.002$ or about $0.1 \%]$.

$\mathrm{ZnS}$, ZnSe, ZnTe, CdTe, and SiC all exhibit polytypism ${ }^{7}$ which is an ordered mixture of $B$ and $W$ stacking of atom planes along the hexagonal $c$ axis. Jagodzinski ${ }^{8}$ has shown on a thermodynamical basis that a necessary condition for polytypism is a lowtemperature cubic phase with $W$-like phases at elevated temperatures. The above five materials must therefore be classified as cubic, which corresponds nicely to their positive $\Delta(c / a)$. The argument can even be made more quantitative. Whereas $\mathrm{ZnS}$ polytypes show no over-all preference in staggered 
(B-like) or eclipsed ( $W$-like) stacking, ${ }^{7}$ staggered SiC polytypes prevail. ${ }^{7}$ Thus SiC should have a larger cubic stability than $\mathrm{ZnS}$ which in fact correlates with a larger $\Delta(c / a)$. On the other hand, the lack of polytypism in CdS and CdSe is evidence supporting the stable $W$ nature of these compounds, and $\Delta(c / a)$ is negative in these cases.

We conclude from this discussion that there is a close empirical correlation between $c / a$ and the $W$-vs- $B$ stability, and that the ideal $c / a=1.633$ is the critical $W$ lattice parameter ratio to within an uncertainty of about $0.1 \%$. One may also notice that the range of $c / a$ values for which the materials are dimorphous (both $W$ and $B$ occur) extends about equally to both sides of the ideal value. This suggests that the relationship between energy difference (stability) and $\Delta(c / a)$ is almost linear.

Our stability considerations did not include BN, $\mathrm{MgS}$, and $\mathrm{MgSe}$ because these materials are actually not believed to be stable in either $W$ or $B$. Thus the stable form of $\mathrm{BN}$ is a graphitelike structure with threefold coordination, and MgS and MgSe normally occur with sixfold coordination in the rocksalt structure.

So far we have ignored the internal distortion described by the deviation $\Delta u$ of the $u$ parameter from its ideal value of 0.375 . It is at once obvious from Table I that $\Delta u$ is known with a reasonable accuracy only for $\mathrm{AgI}, \mathrm{BeO}, \mathrm{ZnO}$, and $\mathrm{AlN}$. Although there is no direct proportionality between $\Delta u$ and $\Delta(c / a)$ (as some authors ${ }^{9}$ have indicated), they do behave similarly; i.e., they are of the same order of magnitude, their signs are consistently opposite, and interpolation indicates that they are zero simultaneously. This feature is actually not very surprising if we compare it with the situation in a $\langle 111\rangle$ uniaxially stressed zinc-blende crystal. Here the relation between the internal and the macroscopic strain is described by the bond-bending parameter ${ }^{10}$ $\zeta$ which is 0.64 in $\mathrm{Ge}$ and $\mathrm{Si}^{11}$ and probably increases with ionicity. ${ }^{12}$ Translated into the $W$ structure from a quasicubic point of view, this gives

$$
\Delta u=-(3 / 128)^{1 / 2} \zeta \Delta(c / a)
$$

and can be fitted to the data in Table $I$ by $\zeta$ in the range $1.6(\mathrm{ZnO})$ to $2.0(\mathrm{AlN}$ and $\mathrm{BeO})$. These rather large values of $\zeta$ may be the reason behind the spectacular piezoelectric constants of AlN and ZnO. ${ }^{13,14}$ The applicability of Eq. (1) with reasonable values of $\zeta^{15}$ shows that the forces causing the $W$ distortion are long range and therefore rather weak, whereas any distortion from the ideal tetrahedral configuration is opposed by strong and mainly short-range forces as described by the elastic constants. A further consequence is that we can neglect the internal strain $\Delta u$ in the following qualitative arguments because $\Delta u$ is determined by $\Delta(c / a)$ through the elasticity forces and would therefore only add irrelevant detail. Note that Keffer and Portis ${ }^{3}$ assumed that $\Delta u$ independently of $\Delta(c / a)$ could be derived from the long-range forces.

We now propose a simple model of the longrange effects accounting for the correlation between stability and distortion $\Delta(c / a)$. For a hypothetical, purely ionic crystal, the Madelung constant for the $B$ structure is 1.6381 (in units referred to the bond length) and $1.6406^{16}$ for the ideal $W$. A distortion with positive $\Delta(c / a)$ and $\Delta u=0$ will lower the Madelung constant as shown by Keffer and Portis ${ }^{3}$ so that $B$ and $W$ energies coincide at a strain determined to first order by

$$
1.6406-0.0207 \Delta(c / a)=1.6381 \text {, }
$$

that is $\Delta(c / a)=+0.12$. This situation is illustrated by curve 1 in Fig. 2 . Since an equilibrium around the ideal configuration is maintained by short-range elasticity forces, the combined effect will be a small negative $\Delta(c / a)$ in this case.

A nonionic crystal will prefer an isotropic arrangement of atoms (with a nearest-neighbor tetrahedral coordination). In $W$, the two third neighbors along the $c$ axis are much closer than any other similar pairs of neighbors in $W$ and $B$ (see Fig. 1). The kinetic energy will then tend to push them apart thus favoring positive distortions $\Delta(c / a)$ as shown in curve 2 in Fig. 2. A positive $\Delta E$ at the ideal structure is also indicated by a recent pseudopotential calculation ${ }^{2}$ of selected valence-band levels in hypothetical, nonionic $W$ and $B$ versions of $\mathrm{ZnS}$.

In Fig. 2, the near coincidence of the crossings of curves 1 and 2 with the zero line is not arbitrary. It will be seen below that there is evidence supporting the feature that the present ionic and nonionic effects have almost the same relative volume dependence. Consequently, it is not farfetched to propose that they also have nearly the same structure dependence apart from a multiplicative factor and opposite sign.

In the intermediate case we add the two curves 1 and 2 with suitable weighting factors because the qualitative features, i.e., the $\Delta E=0$ crossings,

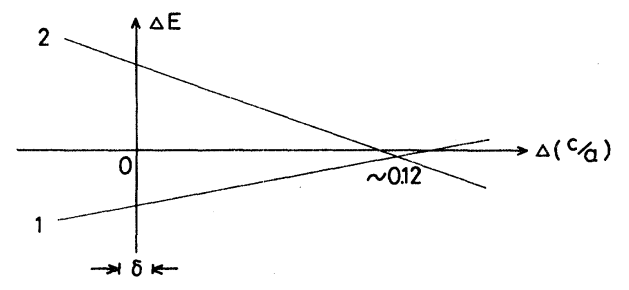

FIG. 2. Difference $\Delta E$ between $W$ and $B$ energy of longrange forces as function of strain $\Delta(c / a)$. Case 1: ionic; case 2 : covalent. The quantity $\delta$ indicates the range of actual distortions $(\sim 1 \%)$. 
are related to the geometry only. It is then obvious from Fig. 2 that we obtain a direct, linear correlation between the energy difference at $\Delta(c / a)=0$ and its derivative at this point, the latter being intimately connected with the resulting strain $\Delta(c / a)$. As the distortion is opposed by elasticity forces, we expect some irregularities in $\Delta(c / a)$ due to material differences in the reduced elastic constants, ${ }^{12}$ but the sign will always give the appropriate stability, $W$ or $B$. We emphasize that the assumption regarding the similar structure dependence of covalent and ionic effects is a necessary condition for the detailed correlation between stability and sign of $\Delta(c / a)$.

It is not surprising that the delicate balance described above may be very sensitive to various crystal imperfections. In CdS, Reynolds et al. ${ }^{17}$ found a strong dependence of $c / a$ on oxygen content, a decrease from 1.624 for the pure crystal to 1.611 at $0.4 \%$ oxygen. Since we predict later that pure CdO should have $c / a=1.610$, this variation can hardly be ascribed to simple alloy features. The result $c / a=1.588$ for $\mathrm{MgS}$ found by Mittendorf $\mathrm{f}^{18}$ is possible evidence for an appreciable influence of thermal stresses on $c / a$.

The above arguments have tacitly assumed that the diatomic volumes of corresponding $W$ and $B$ phases are identical. For dimorphous materials in which both cubic and hexagonal lattice parameters have been measured, it is found that the $W$ phase generally has the larger volume, the difference ranging from $0.06 \%$ in $\mathrm{ZnS}$ to $1.4 \%$ in CdSe. ${ }^{19}$ Small differences $(0.1 \%)$ of opposite sign occur only in $\mathrm{CdTe}$ and ZnSe. It is clear that this effect could easily upset the stability arguments above if the volume dependence of covalent and ionic effects did not match. However, a recent analysis of the elastic constant ${ }^{12}$ shows that this matching is indeed a good approximation there, and so we feel confident that it is in general valid here also. One may also note that the $c / a$ ratio shows very little temperature dependence ${ }^{20}$ thus supporting the above volume independence. On the other hand, we cannot offer a satisfactory explanation for the volume difference which exhibits no definite trends.

In the present model we have only considered the low-temperature energy difference between $W$ and $B$. Thus the model does not provide any explanation for the observed high-temperature $W$-like phases of the otherwise stable cubic materials, e.g., ZnS and SiC. The $c / a$ ratio has been measured for $\mathrm{ZnS}$ as function of temperature $\mathrm{e}^{21}$ up to the $B-W$ transition temperature of $1020^{\circ} \mathrm{C}$. Although it varies in the direction of the ideal ratio, $\Delta(c / a)$ has only decreased by $50 \%$ at the transition. This phase transition is therefore a consequence of another mechanism, and it is suggested that this can be identified as differences in the vibrational part of the free energy. It can be shown that high temperatures will favor the structure which is mechanically softer, but it remains to be shown that $W$ is generally softer than $B$.

\section{RELATION BETWEEN DISTORTION AND IONICITY PARAMETERS}

In Sec. II, we have established that $\Delta(c / a)$ is a quantitative measure of the wurtzite-vs-zinc-blende stability. In the sense that $\Delta(c / a)$ is only a symptom depending on the existence of the $W$ phase, it would be convenient if $\Delta(c / a)$ can be related to an essentially structure-independent quantity. For the $A^{N} B^{8-N}$ compounds, such quantities have recently been produced by the semiempirical dielectric ionicity theory by Phillips ${ }^{1}$ and Van Vechten, ${ }^{2}$ and the success of these concepts applied to an increasing number of phenomena suggests that they might be useful here also.

It was shown above that the distortion is strongly related to long-range Coulomb effects, the covalent contribution being essentially independent of material. Some correlation between $c / a$ and the square of an effective charge parameter is therefore expected. In a recent study of the effective dynamical charges, ${ }^{22}$ the author has related the Szigeti charge $e_{s}^{*}$ to $Z C / \hbar \omega_{p}$, where $Z$ is the (effective) valence, $C$ is Phillips's electronegativity difference, and $\hbar \omega_{p}$ is the plasma energy of the valence-electron gas. This suggests $\left(Z C / \hbar \omega_{p}\right)$ as a relevant charge parameter. In Table $I I$ we have listed $C$ values for a number of materials including those for which the subsequent analysis indicates the possibility of a $W$ phase. Since accurate values of $C$ turn out to be very critical, we have changed some of the more uncertain values ${ }^{23}$ as discussed in the Appendix. It should be stressed that this reexamination does not involve the results to follow.

A plot of available $c / a$ values (Table I) vs the square of $Z C / \hbar \omega_{p}$ (Table II) is shown in Fig. 3 . Except for the first-row compounds $\mathrm{BN}$ and $\mathrm{BeO}$, the diagram points to a detailed linear relationship. In general, if the ionicity at all favors a tetrahedral coordination, we conclude that compounds with $Z C / \hbar \omega_{p}>0.74 \pm 0.01$ will be stable in the $W$ structure, otherwise in the $B$ structure.

The small deviations observed may be ascribed to uncertainties in $c / a$ and $C$ or to differences in the reduced elastic constants related to varying ionicity. ${ }^{12}$

The anomalous behavior of $\mathrm{BN}$ and $\mathrm{BeO}$ is due to the special covalent properties of first-row compounds seen also in the elastic constants of diamond $^{12}$ and in the tendency to form graphitelike phases. For a purely covalent compound such special effects should cancel. If we extrapolate the correlation line in Fig. 3 to $C=0$, we can in fact draw a line from this point through $\mathrm{BN}$ and $\mathrm{BeO}$. 
TABLE II. Ionicity parameters for existing and prospective wurtzite compounds. The electronegativity $C$ is taken from Ref. 23 unless otherwise indicated.

\begin{tabular}{llcc}
\hline \hline Crystal & $Z$ & $C(\mathrm{eV})$ & $Z C / \hbar \omega_{p}{ }^{\mathrm{a}}$ \\
\hline $\mathrm{BeO}$ & 2 & 13.9 & 0.97 \\
$\mathrm{ZnO}$ & 2 & $10.2^{\mathrm{b}}$ & 0.95 \\
$\mathrm{AlN}$ & 3 & 7.30 & 0.95 \\
$\mathrm{CdO}$ & 2 & 9.15 & 0.90 \\
$\mathrm{InN}$ & 3 & $5.46^{\mathrm{b}}$ & 0.87 \\
$\mathrm{GaN}$ & 3 & $6.2^{\mathrm{b}}$ & 0.84 \\
$\mathrm{MgS}$ & 2 & 7.10 & 0.81 \\
$\mathrm{CdS}$ & 2 & 5.90 & 0.80 \\
$\mathrm{MgTe}$ & 2 & $5.10^{\mathrm{b}}$ & 0.78 \\
$\mathrm{MgSe}$ & 2 & 6.41 & 0.78 \\
$\mathrm{CdSe}$ & 2 & $5.40^{\mathrm{b}}$ & 0.77 \\
$\mathrm{BN}$ & 3 & 7.71 & 0.76 \\
$\mathrm{ZnS}$ & 2 & 6.20 & 0.74 \\
$\mathrm{CuF}$ & $1.20^{\mathrm{c}}$ & $14.7^{\mathrm{b}}$ & 0.74 \\
$\mathrm{HgS}$ & 2 & $5.50^{\mathrm{b}}$ & 0.74 \\
$\mathrm{AgI}$ & $1.65^{\mathrm{d}}$ & 5.70 & 0.73 \\
$\mathrm{ZnSe}$ & 2 & 5.60 & 0.72 \\
$\mathrm{CdTe}$ & 2 & 4.40 & 0.71 \\
$\mathrm{HgSe}$ & 2 & $5.00^{\mathrm{e}}$ & 0.71 \\
$\mathrm{CuBr}$ & $1.50^{\mathrm{d}}$ & 6.90 & 0.68 \\
$\mathrm{SiC}$ & 4 & 3.85 & 0.67 \\
$\mathrm{CuCl}$ & $1.35^{\mathrm{d}}$ & 8.30 & 0.67 \\
$\mathrm{InP}$ & 3 & 3.34 & 0.67 \\
$\mathrm{ZnTe}$ & 2 & 4.48 & 0.64 \\
$\mathrm{CuI}$ & $1.65^{\mathrm{d}}$ & 5.50 & 0.64 \\
$\mathrm{HgTe}$ & 2 & $4.00^{\mathrm{e}}$ & 0.62 \\
\hline \hline
\end{tabular}

${ }^{\mathrm{a}} \hbar \omega_{p}$ is calculated from the diatomic volume of the structure for which $C$ is determined (see Ref. 23).

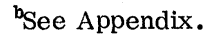

${ }^{c}$ Extrapolated from $\mathrm{CuI}, \mathrm{CuBr}$, and $\mathrm{CuCl}$.

${ }^{d}$ Fitted to the correlation in Fig. 3.

'See Ref. 27.

Since the data for BN have been obtained under rather special circumstances, ${ }^{24}$ we can only say that experiments are not inconsistent with this idea.

For the $\mathrm{Cu}$ and $\mathrm{Ag}$ halides, we have adjusted the effective valence $Z$ to values near 1.5 as shown in Table II. This is a consequence of $d$ electrons. ${ }^{22,23}$ The exact value is rather arbitrary and has in fact been fitted to the curve in Fig. 3, but it is gratifying to notice that only $10 \%$ deviations from 1.5 are needed and that there is a consistent trend with anion species. Some of this variation may actually be the result of very soft elastic behavior as observed in CuCl. ${ }^{12}$ The strain would thus be higher than predicted, corresponding to a smaller adjusted $Z$.

It should also be mentioned that the values of $\left(Z C / \hbar \omega_{p}\right)$ for $\mathrm{MgS}$ and $\mathrm{MgSe}$ are indeed derived from the rocksalt modification of these compounds in which the bond length is about $8 \%$ larger than for $W$ as a consequence of the difference in coordination. If the ionicity $f_{i}$ is invariant under the transformation (as implied by the existence of a critical ionicity), it can be shown that $C / \hbar \omega_{p}$ is about $3 \%$ higher for the $W$ phase. This small correction improves the correlation in Fig. 3 and illustrates the sensitivity to a small change in the choice of parameters.

The most serious deviation left is that of $\mathrm{ZnS}$ where $c / a$ is about $0.2 \%$ larger than expected from the value of $Z C / \hbar \omega_{p}$. According to Fig. 3, we predict the $W$ and $B$ phases to be almost degenerate in energy (which is very appropriate since real wurtzite and zinc blende are actually the two modifications of $\mathrm{ZnS}$ ). This limiting case is therefore very susceptible to a slight perturbation of the basic assumption behind our model in Sec. II, i.e., that the zeros of the ionic (1) and covalent (2) curves in Fig. 2 coincide.

Finally we use the observed correlation between stability and $Z C / \hbar \omega_{p}$ to investigate whether there might be stable wurtzite materials among the $A^{N} B^{8-N}$ compounds which for some reason or other have hitherto escaped observation. It turns out, however, that the only candidate having the ionicity $f_{i}<0.79$ and $Z C / \hbar \omega_{p}>0.74$ is CdO with $f_{i}=0.785^{23}$ and nor mally stable in the rocksalt structure. Like MgS and $\mathrm{MgSe}$, it may show up in a metastable wurtzite phase. Other candidates with $0.60<Z C / \hbar \omega_{p}<0.74$ are $\mathrm{HgS}$, $\mathrm{HgSe}, \mathrm{HgTe}$, In $\mathrm{P}$, and $\mathrm{CuF}$ as shown at. the base line of Fig. 3, but in accordance with our prediction they have so far only occurred in the zinc-blende structure. Our classification of tetrahedrally coordinated $A^{N} B^{8-N}$ compounds in terms of the parameter $Z C / \hbar \omega_{p}$ is thus complete apart from the first-row compounds $\mathrm{BN}$ and $\mathrm{BeO}$ with reasonably understood anomalies.

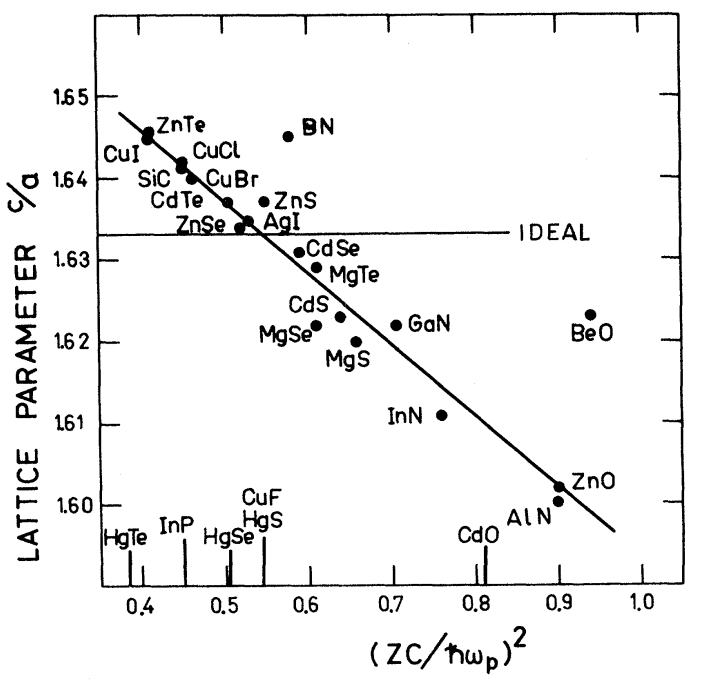

FIG. 3. Correlation between the distortion $(c / a)$ from ideal wurtzite $(c / a=1.633)$ and the square of the ionicity charge parameter $Z C / \hbar \omega_{p}$. The position of prospective candidates for a $W$ phase is shown at the base line. 


\section{CONCLUSION}

The lack of detailed knowledge about Coulomb effects in partially ionic materials prevents a completely satisfactory explanation of the empirical relation between the distortion in $c / a$ and the square of the charge parameter $Z C / \hbar \omega_{p}$. Thus the rigidion model used in Sec. II has serious limitations, and one should certainly expect some sort of screening in this long-range picture. However, it is obvious from Fig. 3 that any attempt to introduce extra correction factors depending on dielectric constant, lattice constant, ionicity, or bond-bending parameter $\zeta$ will have a disastrous effect on the correlation. Somehow, these phenomena are apparently included.

It follows from this conclusion that the key feature of the wurtzite stability and distortion may be visualized as a Coulomb interaction between rigid charges $\pm Z e$ screened by an effective dielectric constant $\epsilon_{\text {eff }}=\left(\hbar \omega_{p}\right)^{2} / C^{2}$. Although this is essentially a picture of the equilibrium, it suggests that the recent ionicity analysis of the dynamic Szigeti charge ${ }^{22}$ should be interpreted along the same lines. However, the local-field problem involved in the Szigeti model will need further examination before a satisfactory solution is reached.

A major result of the present work is the confirmation of $C$ as a significant physical quantity in such a way that an accurate determination of $C$ from the electronic low-frequency dielectric constant $^{1,23}$ leads to a prediction of a lattice parameter, i. e., the wurtzite $c / a$ ratio, to within one part in a thousand. The electronegativity difference $C$ has thereby lost most of its original character as a mere fitting parameter.

\section{APPENDIX}

It is apparent from the results of Sec. III that the values of the electronegativity difference $C$ must be accurate to within a few percent if the correlation between $Z C / \hbar \omega_{p}$ and $c / a$ should be useful. The empirical values of $C$ have been determined from experimental data on $\epsilon(0),{ }^{23}$ the low-frequency electronic dielectric constant. In a few cases, the original determination ${ }^{23}$ was based on questionable data or inferred by extrapolation, and so the present demand for accuracy has led to a reexamination of these values for a number of materials, in particular $\mathrm{ZnO}, \mathrm{GaN}, \mathrm{MgTe}, \mathrm{InN}, \mathrm{CuF}$, and $\mathrm{HgS}$. At an early stage ${ }^{23}$ it was observed that the obtained values of $C$ could be fitted by the following expression (the notation is essentially that of Ref. 23 except for the electronic charge $e$ ):

$$
C=b e^{2}\left(Z_{\alpha} / r_{\alpha}-Z_{\beta} / r_{\beta}\right) e^{-k} s^{R},
$$

where the dimensionless parameter $b$ is very close to 1.50 for most tetrahedrally coordinated com pounds. ${ }^{23}$ Apparently, it has passed unnoticed that actual deviations of $b$ from 1.50 are correlated with the "skewness" of the $A B$ compound, i. e., the row difference between $A$ and $B$ atom. Although this correlation is hard to make quantitative, it should be kept in mind either when experimentally deduced $C$ values are checked on Eq. (A1) or when $C$ is calculated from this expression.

For materials with relatively low $\epsilon(0)$, even a modest change in this quantity may have strong influence on $C$. In $\mathrm{ZnO}$, we replace Van Vechten's choice $^{23}$ of $\epsilon(0)=4.0$ with a more recent value ${ }^{25}$ of 3.75 , thus increasing $C$ from 9.3 to $10.2 \mathrm{eV}$. The corresponding revised value of $b$ is 1.21. A similar analysis for GaN with the experimental value of $\epsilon(0)=5.8^{26}$ yields $C=6.20 \mathrm{eV}$ and $b=1.06$. These $b$ values agree with $b=1.05$ in CdO. ${ }^{23}$ Following these trends we assume $b=1.21$ in CuF (Ref. 23 uses $b=1.30$ ) and obtain $C=14.7 \mathrm{eV}$. For InN we choose $b=1.05$ as in $\mathrm{CdO}$ and so $C=5.46 \mathrm{eV}$ (by scaling the result in Ref. 23 with the new $b$ ). The revised results are listed in Table II.

$\mathrm{MgTe}$ has the opposite type of skewness so that $b$ should be larger than 1.5 (e.g., AlSb has $b \approx 3.0$ ). A comparison of trends in $C$ values in the $\mathrm{Cd}, \mathrm{Zn}$, and $\mathrm{Mg}$ chalcogenides indicates a value of $C=5.10$ $\mathrm{eV}$ for $\mathrm{MgTe}$, corresponding to $b=2.13$.

For HgS, Phillips and Van Vechten ${ }^{27}$ deduced $C=7.3 \mathrm{eV}$ from the observation that the normal cinnabar structure is a distorted version of the rocksalt structure, and since $\mathrm{HgS}$ is also known in the zinc-blende modification, it should have the critical ionicity $f_{i}=0.785$. Actually, the cinnabar structure bears little resemblance to rocksalt, ${ }^{28}$ the coordination number being more like 3 in the former. This is corroborated by a reduction in bond length from zinc-blende $\mathrm{HgS}$ to cinnabar. ${ }^{28}$ We find that $C=5.50 \mathrm{eV}$ is more in accordance with trends among the chalcogenides, and there is no reason to expect serious anomalies.

Finally, a small correction for CdSe is indicated by trends in the chalcogenides. Instead of $C=5.50$ $\mathrm{eV}^{23}$ we use $C=5.40 \mathrm{eV}$. Such details are of course rather questionable, and we have found no reason to apply any correction to $\mathrm{ZnS}$ although this might perhaps remove the worst deviation in Fig. 3.

\footnotetext{
1J. C. Phillips, Rev. Mod. Phys. 42, 317 (1970).

${ }^{2}$ J. A. Van Vechten, Phys. Rev. 187, 1007 (1969).

${ }^{3}$ F. Keffer and A. M. Portis, J. Chem. Phys. 27, 675
}

(1957).

${ }^{4}$ G. A. Jeffrey, G. S. Parry, and R. L. Mozzi, J. Chem. Phys. 25, 1024 (1956). 
${ }^{5}$ J. Krug and L. Sieg, Z. Naturforsch. $\underline{7 a}, 369$ (1952).

${ }^{6}$ M. R. Lorenz and J. S. Prener, Acta Cryst. $\underline{9}, 538$ (1956).

${ }^{7}$ G. C. Trigunayat and G. K. Chadha, Phys. Status Solidi 4 , 9 (1971); G. C. Trigunayat, ibid. 4, 281 (1971).

${ }^{8} \mathrm{H}$. Jagodzinski, Neues Jahrb. Mineral. 10, 49 (1954); H. Jagodzinski and H. Arnold, Proceedings of the Conference on Silicon Carbide, Boston, 1959 (Pergamon, New York, 1960), p. 141.

${ }^{9}$ G. Burley, J. Chem. Phys. 38, 2807 (1963).

${ }^{10}$ L. Kleinman, Phys. Rev. $\underline{128}, 2614$ (1962).

${ }^{11}$ A. Segmüller and H. R. Neyer, Physik Kondensierten Materie $\underline{4}, 63$ (1965).

${ }^{12}$ R. M. Martin, Phys. Rev. B 1 , 4005 (1970).

${ }^{13} \mathrm{~J}$. C. Phillips and J. A. Van Vechten, Phys. Rev. Letters 23, 1115 (1969).

${ }^{14}$ R. M. Martin, Phys. Rev. B (to be published).

${ }^{15}$ It has been suggested earlier that $\zeta>1$ is unphysical, but this is only relevant for very short-range interactions as in nearly covalent materials.

${ }^{16}$ There seems to be some uncertainty as to the exact result. The most recent is 1.64132 [Y. Sakamoto, J. Chem. Phys. 28, 164 (1958) ]. For reasons of consistency, we have used the value computed in Ref. 3 . The exact numbers are not important in the present context.
${ }^{17}$ D. C. Reynolds, S. J. Czyzak, R. C. Allen, and C. C. Reynolds, J. Opt. Soc. Am. 45, 136 (1955).

${ }^{18} \mathrm{H}$. Mittendorf, Z. Physik 183, 113 (1965).

${ }^{19} \mathrm{The} \mathrm{Cu}$ and $\mathrm{Ag}$ halides have been excluded from these considerations because the volume differences may be the result of thermal expansion, the two phases being measured at different temperatures.

${ }^{20}$ R. R. Reeber and B. A. Kulp, Trans. AIME $\underline{233}, 698$ (1965)。

${ }^{21}$ J. G. White and S. G. Ellis (unpublished); see W. B. Pearson, A Handbook of Lattice Spacings and Structures of Metals (Pergamon, New York, 1967), Vol. 2, p. 1228.

${ }^{22}$ P. Lawaetz, Phys. Rev. Letters 26, 697 (1971).

${ }^{23} \mathrm{~J}$. A. Van Vechten, Phys. Rev. 182, 891 (1969).

${ }^{24}$ F. P. Bundy and R. H. Wentorf, J. Chem. Phys. $\underline{38}$, 1144 (1963).

${ }^{25}$ E. C. Heltemes and H. L. Swinney, J. Appl. Phys。 38, 2387 (1967).

${ }^{26}$ D. D. Manchon, A. S. Barker, P. J. Dean, and R. B. Zetterstrom, Solid State Commun. $\underline{8}, 1227$ (1970). ${ }^{27} \mathrm{~J}$. C. Phillips and J. A. Van Vechten, Phys. Rev. B 2,2147 (1970).

${ }^{\frac{28}{28}}$ R. W. G. Wyckoff, Crystal Structures (Wiley, New 'York, 1963), Vol. I, p. 98. 